\section{Is spinal osteophytosis associated with fatty liver?}

\author{
Alexander P. Rozin, ${ }^{1}$ Diana Gaitini, ${ }^{2}$ \\ Kohava Toledano, ${ }^{1}$ \\ Alexandra Balbir-Gurman' \\ 'B. Shine Rheumatology Unit, \\ 2Department of Medical Imaging, \\ Rambam Health Care Campus and \\ Technion, Haifa, Israel
}

\section{Abstract}

Lipopolysaccharide (LPS) is a bacterial endotoxin. LPS-induced cartilage damage is widely used as a model to investigate the effectiveness of cartilage protective agents in vitro. Overwhelming liver LPS of intestinal origin break impaired liver barriers, achieve blood stream in low but sustained accounts and spread to joints. That results in LPS-binding protein (LBP) - CD14 activation of inflammatory response and development of degenerative joints changes (osteophyte formation and cartilage loss). Easy penetration of LPS into circulation may be a result from liver dysfunction associated with fatty liver. We proposed an analogical in vivo model of bone degenerative changes related to fatty liver. Ultrasound (US) images of patients admitted to the emergency department for abdominal pain were revised. Fifty two patients were divided to two groups. The first included 26 patients ( $M / F=13 / 13$, age of $56.2 \pm 8.5$ years) with US features of fatty liver. The second comprised of 26 patients $(\mathrm{M} / \mathrm{F}=13 / 13$, age of $57.5 \pm 8.0$ years, $\mathrm{P}=0.56)$ without US features of fatty liver. Retrospective analysis of images of the whole spine (X-ray, computed tomography) in both populations obtained from the hospital imaging data base, was performed. Four maximal size spinal osteophytes were measured. In order to reduce an influence of individual body size factors the spine osteophyte radial length/vertebral width $(0 / N)$ ratio was calculated. Hence, the osteophyte size was measured as a part of a vertebral width. Correlation between osteophytes' size, patient age, and body mass index (BMI) in both groups was assessed. Spine osteophytes of patients with fatty liver had a bigger size $(0 / N=0.21 \pm 0.06)$ compared with patients with normal liver $(0 / \mathrm{V}=0.14 \pm 0.03, \mathrm{P}<0.001)$. Fatty liver patients had a higher BMI $\left(32.7 \pm 5.4 \mathrm{~kg} / \mathrm{m}^{2}\right)$ compared to normal liver patients $\left(28.0 \pm 6.7 \mathrm{~kg} / \mathrm{m}^{2}\right.$, $\mathrm{P}=0.031$ ). Spine with normal liver had marginal vertebral bulging less 0.17 or $17 \%$ of the vertebral width. Maximal osteophyte size was 0.3 of the vertebral width or $30 \%$ in fatty liver group. In order to distinguish an influence of BMI and fatty liver on $\mathrm{O} /$, patients of normal liver group were divided according to increased BMI $\left(>25 \mathrm{~kg} / \mathrm{m}^{2}\right)$ and normal one $\left(<25 \mathrm{~kg} / \mathrm{m}^{2}\right) .0 / \mathrm{N}$ of both subgroups showed similar values: $0.14 \pm 0.03$ (high BMI) and $0.13 \pm 0.02$ (normal $\mathrm{BMI})(\mathrm{P}=0.52)$. BMI of the subgroups was significantly different: $31.8 \pm 5.4$ (high BMI) and 21.3 \pm 1.7 (normal BMI) $(\mathrm{P}<0.001)$. No direct correlation was found between $\mathrm{O} / \mathrm{N}$ and BMI in fatty liver and normal liver groups ( $\mathrm{P}=0.81, \mathrm{P}=0.51$ respectively). In opposite, significant $\mathrm{O} / \mathrm{N}$ and age correlation was found in normal liver group $(\mathrm{P}=0.014)$ and weak correlation in fatty liver group $(\mathrm{P}=0.063)$. Spine osteophytes of patients with fatty liver have a bigger size compared with patients with normal liver. Fatty liver may be considered as a predisposing factor in the pathogenesis of spine osteophytosis.

\section{Introduction}

Osteoarthritis (OA, also known as degenerative arthritis, degenerative joint disease, DJD), is a clinical syndrome, in which low grade inflammation and abnormal wearing of the cartilage, affecting lubricating properties of the synovial fluid, results in joint pain and disability due to cartilage damage, osteophyte formation, bone and soft tissue periarticular disease. Previously assessed as a secondary process to mechanical problems or inflammatory joint diseases s, low grade inflammation is considered today as an important factor in pathogenesis. ${ }^{1} 0 \mathrm{~A}$ is the most common form of arthritis. It may rapidly cause joint destruction and is the leading cause of chronic disability. ${ }^{2}$ OA affects nearly $7 \%$ of the population and accounts for $25 \%$ of visits to primary care physicians, and half of all NSAID (NonSteroidal Anti-Inflammatory Drugs) prescriptions. It is estimated that $80 \%$ of the population will have radiographic evidence of $0 \mathrm{~A}$ by age 65 , although only $60 \%$ of them will be symptomatic. In the United States, hospitalizations for osteoarthritis soared from approximately 322000 in 1993 to 735000 in 2006 . The main pathological feature of $\mathrm{OA}$ is abnormal bone (osteophyte) formation, subchondral bone sclerosis, and cartilage loss). This is governed by members of the transforming growth factor/bone morphogenetic protein protein family, as well as by the group of Wingless (Wnt) proteins. Wnt proteins seem to be regulators of osteophyte formation. Binding of Wnt to its receptor Lrp5/6 and activation of downstream regulators such as beta-catenin are crucial steps for bone formation. ${ }^{3}$ Wnt activation also blocks osteoclast differentiation through increased production of osteoprotegerin. ${ }^{4} \mathrm{~A}$ functional relationship between Wntinduced secreted protein-1 (WISP-1) and transforming growth factor (TGF) $\beta$ as modu-
Correspondence: Alexander P. Rozin, Department of Rheumatology, Rambam Health Care Campus, POB 9602, Haifa 31096, Israel.

Tel. +972.4 .8542268 - Fax: +972.4 .8542985$

E-mail: a_rozin@rambam.health.gov.il

Key words: osteophyte, fatty liver, lipopolysaccharide, inflammatory activation- LPS-LBP-CD14.

Conflict of interest: the authors declare no conflict of interests.

Received for publication: 30 November 2011.

Revision received: 23 January 2012.

Accepted for publication: 7 February 2012.

This work is licensed under a Creative Commons Attribution NonCommercial 3.0 License (CC BYNC 3.0).

(C) Copyright A.P. Rozin et al., 2012

Licensee PAGEPress, Italy

Rheumatology Reports 2012; 4:e4

doi:10.4081/rr.2012.e4

lators of proliferation and osteogenic differentiation has recently been found. ${ }^{5}$ WISP-1 may be a novel regulator of TGF- $\beta 1$ signaling in osteoblast-like cells.

Lipopolysaccharide (LPS) is bacterial endotoxin. LPS-induced cartilage damage is widely used as a model to investigate the effectiveness of cartilage protective agents in vitro. ${ }^{6-8}$ We proposed an analogical in vivo model of joint degenerative changes related to fatty liver. Might similar LPS-induced cartilage damage occur in vivo according to scenario such as follows? Overwhelming liver LPS of intestinal origin break vulnerable liver barriers of fatty liver, achieve blood stream in low but sustained accounts, and spread to joints. This result in LPS-lipopolysaccharide-binding protein (LBP)-CD14 activation of inflammatory response associated with enhanced synthesis of interleukins (IL) IL-1, IL-6, tumor necrosis factor (TNF)- $\alpha$, interferon (INF) INF- $\gamma$, nitric oxide (NO), TGF- $\beta$ and development of degenerative joint changes.

As well obesity is nowadays considered as a chronic low-grade inflammatory condition, which is closely related to the release of multiple factors of proinflammatory nature by white adipose tissue. They include classical cytokines such as IL-6, IL-1 and TNF- $\alpha$, as well as adipokines, such as leptin, adiponectin, resistin, visfatin. Other factors such as chemerin, lipocalin, or serum Amiloyd 3 (SAA3) have recently been identified.9,10 That is why along with increased mechanical load osteoarthritic cartilage may be affected by biologically active blood constituents.

Fatty liver results from an increased fatty acid uptake, decreased low density lipoproteins export, and impaired lipid metabolism. 
There are several liver conditions associated with superfluous fat deposits. ${ }^{11}$ The first is non-inflammatory steatosis affects approximately $25-35 \%$ of the general population. The last may further transform to inflammatory steatohepatitis, which may be alcohol induced or unrelated to alcohol consumption (NASHnon-alcoholic steatohepatitis). NASH has been detected in $1.2-9 \%$ of patients undergoing routine liver biopsy, and in over $80 \%$ of patients who are obese. Over $50 \%$ of patients undergoing bariatric surgery have NASH. Fibrosis or cirrhosis in the liver develops in $15-50 \%$ of patients with NASH. There is no doubt that NASH is a part of systemic disease such as metabolic syndrome associated with liver damage. Persistent fatigue and malaise were reported as main extrahepatic manifestations of fatty liver. We proposed one more: accelerated spine osteophyte formation.

The aim of this study is to assess the size of spinal osteophytes in patients with fatty liver in comparison to spinal osteophytes in normal liver control group.

\section{Materials and Methods}

Retrospective analysis of whole spine X-rays and/or computed tomography (CT), of patients (pts) who underwent abdominal ultrasound (US) while visited the emergency department for different reasons was performed. Patients' data with their diseases and medications is presented on Table 1. The images were obtained from the hospital imaging database. Fifty two patients were divided to two groups (Table 2). The first included 26 patients (M/F $=13 / 13$, age of $56.2 \pm 8.5$ years) with US features of fatty liver. The second comprised of 26 patients $(M / F=13 / 13$, age of $57.5 \pm 8.0$ years, $\mathrm{P}=0.56$ ) without US features of fatty liver. Patient height and weight data were collected from medical records, Body Mass Index (BMI) was calculated. Retrospective analysis of whole spine X-ray and/or CT in both groups was performed, and four maximal size spinal osteophytes were measured. In order to reduce an influence of individual body size factors the spine osteophyte radial length/vertebral width $(0 / \mathrm{V})$ ratio was calculated (Figure 1$)$. Hence, the osteophyte size was measured as a part of a vertebral width. Correlation between osteophytes size, patient age, and BMI in both subgroups was assessed. Medical records were reviewed. The patients did not have complaints of musculoskeletal disorders.

\section{Exclusion criteria}

Cases of patients with diffuse idiopathic skeletal hyperostosis, history of retinoid therapy, history or imaging features of metabolic bone diseases (osteoporosis, osteopetrosis, chondrocalcinosis), bone fractures or metastasis, history or imaging features of spondyloarthropathies, history of chronic liver diseases (liver cirrhosis, viral hepatitis, hemochromatosis, Wilson disease, autoimmune hepatitis), history of decompensated heart, lung, and kidney disease, central nervous system diseases, history of hepatotoxic medications or corticosteroids use were not included in the analysis.

\section{Statistical analysis}

Data were analyzed using SPSS 12.0 software. Comparison between continuous variables of SSPR was done with the MannWhitney U non-parametric test. For group comparison of age variables a two-tailed Student's t-test was used. Spearman's non-parametric
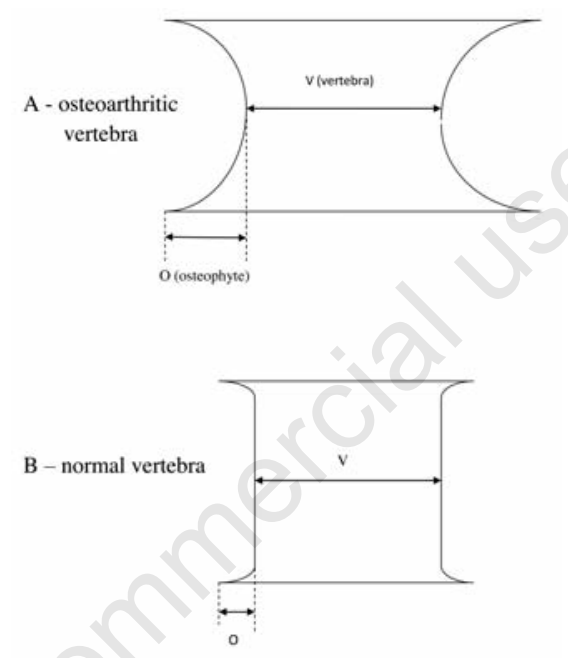

Figure 1. Marginal bulging of vertebra osteophyte $(\mathrm{O})$ was measured and divided to vertebral width $(\mathrm{V})$ in order to get $\mathrm{O} / \mathrm{V}$ ratio for assessment of the osteophyte size. correlation was performed in order to examine relationships between age, osteophyte size and BMI. P values of 0.05 or less were considered as statistically significant.

\section{Results}

Spine osteophytes of patients with fatty liver had $50 \%$ bigger size $(0 / N=0.21 \pm 0.06)$ compared with patients with normal liver $(\mathrm{O} / \mathrm{V}=0.14 \pm 0.03, \quad \mathrm{P}<0.0001) \quad($ Table 2). Maximal osteophyte size was 0.3 of the vertebral width or $30 \%$ in fatty liver group. Spine with normal liver had marginal vertebral bulging less 0.17 or $17 \%$ of the vertebral width (Table 1, Figure 1). Fatty liver patients had a

Table 1. Data of patients' diseases and medications.

\begin{tabular}{lcc}
$\begin{array}{l}\text { Patients' } \\
\text { diseases } \\
\text { and } \\
\text { medications }\end{array}$ & $\begin{array}{r}\text { Fatty liver } \\
\text { N. of } \\
\text { xpatients }\end{array}$ & $\begin{array}{c}\text { Normal liver } \\
\text { N. of } \\
\text { patients }\end{array}$ \\
$\begin{array}{l}\text { Biliary colic } \\
\text { Peptic disease }\end{array}$ & 3 & 2 \\
\hline $\begin{array}{l}\text { Acute gastritis } \\
\text { Irritable bowel syndrome }\end{array}$ & 2 & 6 \\
\hline $\begin{array}{l}\text { Undifferentiated } \\
\text { abdominal pain }\end{array}$ & 12 & 11
\end{tabular}

\begin{tabular}{lcc} 
Renal colic & 2 & 1 \\
\hline Arterial hypertension & 3 & 2 \\
Obesity (body mass & 26 & 13 \\
index $>25 \mathrm{~kg} / \mathrm{m}^{2}$ ) & & \\
\hline Statines & 3 & 4 \\
Beta-blockers & 3 & 2 \\
\hline Angiotensin convertin & 3 & 1
\end{tabular}

enzyme inhibitors

Proton pump inhibitors 4

Table 2. Data of age, body mass index, osteophyte radial length/vertebral width-body mass index-age correlations in fatty liver and normal liver groups.

\begin{tabular}{llll} 
Variable & $\begin{array}{l}\text { Patients } \\
\text { fatty liver (26) }\end{array}$ & $\begin{array}{l}\text { Patients with } \\
\text { with N liver }(26)\end{array}$ & $\mathrm{P}$ \\
Age, years & $56.15 \pm 8.5$ & $57.5 \pm 8.0$ & $\mathrm{P}=0.56$ \\
$\mathrm{BMI}, \mathrm{kg} / \mathrm{m}^{2}$ & $32.7 \pm 5.4$ & $28.0 \pm 6.7$ & $\mathrm{P}=0.031$ \\
\hline $\mathrm{O} N$ ratio & $0.21 \pm 0.06$ & $0.14 \pm 0.03$ & $\mathrm{P}<0.001$ \\
Correlation ON:BMI & -0.06 & 0.16 & $\mathrm{P}=0.81, \mathrm{P}=0.51$ \\
\hline Correlation $\mathrm{O} / \mathrm{Age}$ & 0.37 & 0.48 & $\mathrm{P}=0.063, \mathrm{P}=0.014$ \\
$\mathrm{O} N$ ratio $\mathrm{BMI}>25 \mathrm{~kg} / \mathrm{m}^{2}$ & $0.21 \pm 0.06(26$ patients) & $0.14 \pm 0.03\left({ }_{13 \text { patients }}\right)$ & $\mathrm{P}=0.52$ \\
$\mathrm{O} N$ ratio $\mathrm{BMI}<25 \mathrm{~kg} / \mathrm{m}^{2}$ & None & $0.13 \pm 0.02\left(_{\text {13patients }}\right)$ & $\mathrm{P}=0.52$ \\
\hline
\end{tabular}

Age and body mass index (BMI) data is presented in lines 1-2. No differences were in age of patients between fatty liver and $\mathrm{N}$ liver group, but BMI was 14\% higher in fatty liver group. Line 3 depicts spine osteophyte size/vertebral body width ration ( $O N$ ratio), which proved to be $50 \%$ higher in fatty liver group compared to patients with normal liver. Correlations OV to BMI were not found in both groups $p>0.05$ (line 4). But line 5 of the table shows correlations of $\mathrm{OV}$ to age, much more convinced in patients with normal liver. No differences in $\mathrm{ON}$ ratio between normal liver patients with increased BMI (13/50\%) and normal BMI (13/50\%) was found (lines 6-7 right side). However, all patients with fatty liver had BMI higher $25 \mathrm{~kg} / \mathrm{m}^{2}$ (line 6 left side). 
higher BMI $\left(32.7 \pm 5.4 \mathrm{~kg} / \mathrm{m}^{2}\right)$ compared to normal liver patients $\left(28.0 \pm 6.7 \mathrm{~kg} / \mathrm{m}^{2}, \mathrm{P}=0.031\right)$. In order to distinguish an influence of BMI and fatty liver on $0 / \mathrm{N}$, patients in normal liver group were divided according to BMI levels: increased (BMI $>25 \mathrm{~kg} / \mathrm{m}^{2}$ ) and normal one ( $<25 \mathrm{~kg} / \mathrm{m} 2) .0 / \mathrm{N}$ of both subgroups showed similar values: $0.14 \pm 0.03\left(\mathrm{BMI}>25 \mathrm{~kg} / \mathrm{m}^{2}\right)$ and $0.13 \pm 0.02\left(\mathrm{BMI}<25 \mathrm{~kg} / \mathrm{m}^{2}\right)(\mathrm{P}=0.52)$ (Table 2). BMI of the subgroups was significantly different: $31.8+/-5.4 \quad(\mathrm{BMI}>25)$ and $21.3 \pm 1.7$ $(\mathrm{BMI}<25) \quad(\mathrm{P}<0.001)$. However, all patients with fatty liver had BMI higher $25 \mathrm{~kg} / \mathrm{m}^{2}$ and there was not subgroup for comparison (Table 2). No direct correlation was found between $\mathrm{O} / \mathrm{V}$ and $\mathrm{BMI}$ in both groups: fatty liver and normal liver $(\mathrm{P}=0.81, \mathrm{P}=0.51$ respectively). In opposite, $\mathrm{O} / \mathrm{N}$ and age significant correlation was found in normal liver group $(\mathrm{P}=0.014)$ and weak correlation in fatty liver group $(\mathrm{P}=0.063)$.

\section{Discussion}

Main results of our trial are two: i. patients with fatty liver asymptomatic from the view of spine complaints had $50 \%$ bigger size of osteophytes compared with normal liver group; ii. subgroup of patients with normal liver and high BMI had similar osteophyte size like that of subgroup of normal liver and normal BMI. No correlation of BMI and osteophyte size was found. We propose fatty liver plays a dominant role in formation of osteoarthritic spine changes by bacterial LPS-mechanism.

Bacterial LPS typically consist of a hydrophobic domain known as lipid A (or endotoxin), a non-repeating core oligosaccharide, and a distal polysaccharide (or 0-antigen). Recent genomic data have facilitated the role of LPS assembly in diverse Gram-negative bacteria, many of which are human or plant pathogens, and have established the importance of lateral gene transfer in generating structural diversity of 0 -antigens. Many enzymes of lipid A biosynthesis (like LpxC) have been validated as targets for development of new antibiotics. Key genes for lipid A biosynthesis have been found in higher plants, indicating that eukaryotic lipid A-like molecules may exist. The plasma membrane protein TLR4 was identified as the lipid A signaling receptor of animal cells. TLR4 belongs to a family of innate immunity receptors that possess a large extracellular domain of leucinerich repeats, a single trans-membrane segment, and a smaller cytoplasmic signaling region that engages the adaptor protein MyD88. The expanding knowledge of TLR4 specificity and its downstream signaling pathways should provide new opportunities for blocking inflammation associated with infec- tion. ${ }^{2}$ LPS dissociated from $E$. coli is bound in liver with LPB and transferred to CD14-receptor of LPS, or to TLR4-MD2 complex distributed on immunocompetent cells. That starts signal transduction, involving MyD88, IRAK-S, TRAF6, NIK, IKK-S with NF-kB translocation into nucleus with pro-inflammatory cytokine response: IL-1, TNF- $\alpha$, co-stimulating molecules B7, tissue factor, and etc. Inflammatory activation induces cartilage damage and bone overgrowth. ${ }^{13,14}$ Recently relationships between major players in this process have been explored. Concentrations of plasma endotoxin, LBP, sCD14, several cytokines, acute phase proteins and clinical activity indices were studied in 104 patients with Crohn's disease (CD) and 52 patients with ulcerative colitis (UC). Endotoxemia was present in $48 \%$ of CD and in $28 \%$ of UC pts. The mean levels of LBP were higher in patients with active CD $(23.1 \pm 13.7$ $\mathrm{mcg} / \mathrm{mL})$ and $\mathrm{UC}(21.4 \pm 10.9 \mathrm{mcg} / \mathrm{mL})$ than in healthy controls $(7.2 \pm 1.8 \mathrm{mcg} / \mathrm{mL} ; \mathrm{P}=0.01)$. Elevated concentrations of endotoxin and LBP were even detected in patients with inactive CD. Higher endotoxin levels correlated with clinical activity scores and the highest LBP levels. Treatment normalized LBP, endotoxin and sCD14 levels. ${ }^{15}$ LBP plasma levels and TNFalpha gene expression on liver biopsy species were investigated in 40 pts with morbid obesity and non-alcoholic fatty liver disease. Endotoxemia assessed by the circulating level of LBP; The expression of TNF- $\alpha$ in liver tissue was evaluated by real-time polymerase change reaction (PCR). Levels of LBP were increased compared to normal-weight controls; plasma levels of LBP were higher in patients with steatohepatitis $(14.2 \pm 3.9 \mu \mathrm{g} / \mathrm{mL})$ when compared to patients with simple steatosis $(11.5 \pm$ $3.2 \mu \mathrm{g} / \mathrm{mL}), P=0.041$. The TNF- $\alpha$ mRNA expression in liver tissue was significantly higher in patients with NASH and correlated with elevated plasma LPB levels ( $\mathrm{r}=0.412$, $\mathrm{P}=0.036$ ). This data support the potential role of endotoxin in the pathogenesis of non-alcoholic steatohepatitis. ${ }^{16}$ Raised TNF- $\alpha$ gene expression in the hepatic tissue supposed as proinflammatory factor, and in turn, may be involved in formation of inflammatory or degenerative bone changes in spine. Our results indicate that pts with fatty liver had significantly bigger osteophytosis than pts without liver changes. In fatty liver group these correlation was not explained by BMI differences.

There is little known regarding LPS, LBP, and CD14 levels in osteoarthritis patients. Levels of endotoxin in the synovial fluid from pts with OA (21 pts) were significantly higher than in pts undergoing hemodialysis (42 pts), and resembled profile in synovial fluid from pts with rheumatoid arthritis (RA, 26 pts): $8.07 \pm 5.04 ; 5.13 \pm 4.72 ; 13.04 \pm 12.26$ correspondently (normal level<10 pg/mL). ${ }^{17}$ LPS were found in peri-prosthetic tissue in one out of six patients after $\mathrm{OA}$ joint replacement and in all 4 patients after RA joint replacement in other trial. ${ }^{18}$

In our study osteophytes with maximal size were observed in thoracic-lumbar region. This closest neighborhood to hepatic veins may explain the spine osteophytes formation. Extended venous bridging between spine venous plexuses and abdominal-pelvic veins is a known structural feature. ${ }^{19}$ LPS are unstable and are found in serum during $5-15 \mathrm{~min}$ only. ${ }^{20,21}$ But even through this short period their biological actions may be maximal on adjacent to liver and intestine taking to account thoracic-lumbar spine by penetration through epi-spine vascular connections.

In opposite to existing view regarding synovitis in OA as secondary disease to mechanical abnormalities, ${ }^{22}$ our approach proposes $\mathrm{OA}$ as primary inflammation results from proposed LPS-LBP-CD14/TLR-4 activation. There are several lines of evidence that origins of $\mathrm{OA}$ should be looked for outside the joints.

Brandt KD suggests that $O A$ is not merely a cartilage disease, but a disorder affects all tissues of the joint: periarticular bursitis, enthesopathy, ligament strain or muscle spasm, synovitis, medullary hypertension in the cancellous subchondral bone or bone marrow lesions. Such multiple target involvement is suggested to be from extraarticular sources disseminating to joints and acting to all joint structures..$^{22}$

Another line of evidence results from strong hormonal involution-OA relationship, which is far from mechanical events. Estrogen and testosterone deficiency reduces resistance of chondrocyte to wide variety of stresses and diminishes regenerative properties of bone and cartilage. ${ }^{23}$

There are findings from daily practice of multiple complaints of patients with $\mathrm{OA}$, including bone and muscle pain, weakness, fatigue, and malaise. These are typical complaints of pts with systemic diseases.

Experimental endotoxemia in humans was also is accompanied by systemic symptoms such as myalgia, arthralgia, and weakness! ${ }^{20}$ Recent data suggest similarity between $\mathrm{OA}$ and RA, the disease with obvious extraarticular B-cell-RF expansion, especially in the view of similar, pro-inflammatory cytokines profile: IL-1, IL-6, TNF- $\alpha$. Synovitis in both diseases is, characterized with mononuclear infiltration and pannus formation. In both diseases there is enhanced activity of matrix metalloproteases. ${ }^{24}$

Most people with OA could not indicate any traumatic episode that could implement development of $\mathrm{OA}$ in specific joint. Mechanical theory of $\mathrm{OA}$ explains only a group of patients with $0 \mathrm{~A}$, which had a history of obvious trauma. Such traumatic event causes intra-articular derangement 
with abnormal tissue formation, which might be an attractive site (nidus) for alien pathogen associated microbial particles and chemotaxis of immune-competent cells.

- Proinflammatory cytokines of the body fat origin and adipokines. ${ }^{25,26}$

\section{Conclusions}

Spine osteophytes of patients with fatty liver have a bigger size compared with patients with normal liver. The size of osteophytes was not correlated with BMI but was related to fatty liver. LPS-LPB-CD14 mechanism of inflammation should be investigated in patients with degenerative spine disease and fatty liver. Fatty liver may be proposed as a predisposing factor in the pathogenesis of spine osteophytosis. Further studies are needed including extended samples of patient groups.

\section{References}

1. Orita S, Koshi T, Mitsuka T, et al. Associations between proinflammatory cytokines in the synovial fluid and radiographic grading and related scores in 47 consecutive patients with osteoarthritis of the knee. BMS Musculoskelet Disord 2011;12:144.

2. DiBonaventura MD, Gupta S, McDonald MM, Sadosky A. Evaluating the health and economic impact of osteoarthritis pain in the workforce: results from the National Healthand Wellness Survey. BMC Musculoskeletal Disord 2011;12:83.

3. Miller JR. The Wnts. Genome Biol 2002 $3: 3001$

4. Lu W, Kim KA, Liu J, et al. R-spondinl synergizes with Wnt3A in inducing osteoblast differentiation and osteoprotegerin expression. FEBS Lett 2008;582:643-50.

5. Inkson CA, Ono M, Kuznettsov SA, et al. TGF-betal and WISP-1/CCN-4 can regulate each other's activity to cooperatively control osteoblast function. J Cell Biochem
2008;104:1865-78.

6. Campo GM, Avenoso A, Campo S, et al. Differential effect of molecular mass hyaluronan on lipopolysaccharide-induced damage in chondrocytes.Innate Immun 2010;16:48-63.

7. Hayashi M, Kadomatsu K, Ishiguro N. Keratan sulfate suppresses cartilage damage and ameliorates inflammation in an experimental mice arthritis model. Biochem Biophys Res Commun 2010;401: 463-8.

8. Oestergaard S, Rasmussen KE, Doyle N, et al. Evaluation of cartilage and bone degradation in a murine collagen antibodyinduced arthritis model. Scand J Immunol 2008;67:304-12.

9. Fantuzzi G. Adiponectin and inflammation: consensus and controversy. J Allergy Clin Immunol 2008;121:326-30.

10. Conde J, Gomez R, Bianco G, et al. Expanding the adipokine network in cartilage: identification and regulation of novel factors in human and murine chondrocytes. Ann Rheum Dis 2011;70:551-9.

11. Torres DM, Jones FJ, Shaw JC, et al. Rosiglitazone versus Rosiglitazone and metformine versus Rosiglitazone and Losartan in the treatment of non-alcoholic steatohepatitis in humans: a 12-month randomized, prospective, open-label trial. Hepatology 2011;54:1631-9.

12. Raetz CR, Whitfield C. Lipopolysaccharide endotoxins. Annu Rev Biochem 2002;71: 635-700.

13. Clockaerts S, Bastaansen-Jenniskens YM, Feijt C, et al. Peroxicome proliferator aftivated receptor alpha activation decreases inflammatory and destructive responses in osteoarthritic cartilage. Osteoarthritis Cartilage 2011;19:895-902.

14. Leistad L, Feuerherm A, Faxvaag A, Johansen B. Multiple phospholipase A2 enzymes participate in the inflammatory process in osteoarthritic cartilage. Scan J Rheumatol 2011;40:308-16.

15. Pastor Rojo 0, Lopez San Roman A, AlbenizArbizu E, et al. Serum lipopolysaccharide-binding protein in endotoxemic patients with inflammatory bowel disease. Inflamm Bowel Dis 2007;13:269-77.

16. Ruiz AG, Casafont F, Crespo J, et al.
Lipopolysaccharide-binding protein plasma levels and liver TNF-alpha gene expression in obese patients: evidence for the potential role of endotoxin in the pathogenesis of non-alcoholic steatohepatitis. Obes Surg 2007;17:1374-80.

17. Shiota E, Maekawa M, Kono T. Analysis of the levels of endotoxin and beta-d- glucan in the synovial fluid of hemodyalysis patients. Mod Rheumatol 2001;11:304-7.

18. Nalepka JL, Lee MJ, Kraay MJ, et al. Lipopolysaccharide found in aseptic loosening of patients with inflammatory arthritis. Clin Orthop Relat Res 2006;451: 229-35.

19. Tobinick E, Vega CP. The cerebrospinal venous system: anatomy, physiology, and clinical implications. MedGenMed 2006;8:53

20. By Sander JH, van Deventer SJ, Buller HR, et al. Experimental endotoxemia in humans: analysis of cytokine release and coagulation, fibrinolytic, and complement pathways. Blood 1990;76:2520-6.

21. Albillos A, de la Nera A, Gonzalez M, et al. Increased lipopolysaccharide binding protein in cirrhotic patients with marked immune and hemodynamic derangement. Hepatology 2003;37:208-17.

22. Brandt KD. Why should we expect a structure-modifying osteoarthritis drug to relieve osteoarthritis pain? Ann Rheum Dis 2011;70:1175-7.

23. Roman-Blas JA, Castañeda S, Largo R, Herrero-Beaumont G. Osteoarthritis associated with estrogen deficiency. Arthritis Res Ther 2009;11:241.

24. Wang HS, Kuo PY, Yang CC, Lyu SR. Matrix metalloprotease-3 expression in the medial plica and pannus-like tissue in knees from patients with medial compartment osteoarthritis. Histopathology 2011;58: 593-600.

25. Guzzardi MA, Iozzo P. Fatty heart, cardiac damage and inflammation. Rev Diabet Stud 2011;8:403-17.

26. Rai MF, Sandell LJ. Inflammatory mediators: tracing links between obesity and osteoarthritis. Crit Rev Eukaryot Gene Expr 2011;21:131-42. 